\title{
BMJ Open New predictive model for microsurgical outcome of intracranial arteriovenous malformations: study protocol
}

\author{
Xianzeng Tong,,$^{1,2,3,4}$ Jun Wu, ${ }^{1,2,3,4}$ Yong Cao, ${ }^{1,2,3,4}$ Yuanli Zhao, ${ }^{1,2,3,4}$ \\ Shuo Wang ${ }^{1,2,3,4}$
}

To cite: Tong $X$, Wu J, Cao $Y$, et al. New predictive model for microsurgical outcome of intracranial arteriovenous malformations: study protocol. BMJ Open 2017;7: 014063. doi:10.1136/bmjopen-2016014063

- Prepublication history for this paper is available online To view these files please visit the journal online (http://dx.doi.org/10.1136/ bmjopen-2016-014063)

Received 29 August 2016 Revised 24 October 2016 Accepted 14 December 2016

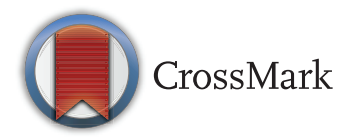

\footnotetext{
${ }^{1}$ Department of Neurosurgery, Beijing Tiantan Hospital, Capital Medical University, Beijing, China ${ }^{2}$ China National Clinical Research Center for Neurological Diseases, Beijing, China

${ }^{3}$ Center of Stroke, Beijing Institute for Brain Disorders, Beijing, China

${ }^{4}$ Beijing Key Laboratory of Translational Medicine for Cerebrovascular Diseases, Beijing, China
}

Correspondence to Professor Shuo Wang; captain9858@vip.sina.com

\section{ABSTRACT}

Introduction: Although microsurgical resection is currently the first-line treatment modality for arteriovenous malformations (AVMs), microsurgery of these lesions is complicated due to the fact that they are very heterogeneous vascular anomalies. The Spetzler-Martin grading system and the supplementary grading system have demonstrated excellent performances in predicting the risk of AVM surgery. However, there are currently no predictive models based on multimodal MRI techniques. The purpose of this study is to propose a predictive model based on multimodal MRI techniques to assess the microsurgical risk of intracranial AVMs.

Methods and analysis: The study consists of 2 parts: the first part is to conduct a single-centre retrospective analysis of 201 eligible patients to create a predictive model of AVM surgery based on multimodal functional MRIs (fMRIs); the second part is to validate the efficacy of the predictive model in a prospective multicentre cohort study of 400 eligible patients. Patient characteristics, AVM features and multimodal fMRI data will be collected. The functional status at pretreatment and 6 months after surgery will be analysed using the modified Rankin Scale (mRS) score. The patients in each part of this study will be dichotomised into 2 groups: those with improved or unchanged functional status (a decreased or unchanged mRS 6 months after surgery) and those with worsened functional status (an increased mRS). The first part will determine the risk factors of worsened functional status after surgery and create a predictive model. The second part will validate the predictive model and then a new AVM grading system will be proposed.

Ethics and dissemination: The study protocol and informed consent form have been reviewed and approved by the Institutional Review Board of Beijing Tiantan Hospital Affiliated to Capital Medical University (KY2016-031-01). The results of this study will be disseminated through printed media.

Trial registration number: NCT02868008.

\section{INTRODUCTION}

The incidence of intracranial arteriovenous malformations (AVMs) is estimated as 1.12-

\section{Strengths and limitations of this study}

- The study is designed to provide a new supplementary grading scale in predicting surgical outcomes of arteriovenous malformations (AVMs) of the brain.

- The new predictive model based on multimodal MRI techniques may be superior to currently existing AVM grading systems.

- Selection bias may exist in the prospective phase of the study that consists of patients treated by different neurosurgeons at different neurosurgical centres.

1.42 cases per 100000 person-years. ${ }^{1}$ The overall annual bleeding rate of untreated AVMs is reported to range from $2.10 \%$ to $4.12 \% .{ }^{1-5}$ Intracranial AVMs are associated with high risk of morbidity and mortality. ${ }^{6-9}$ Current treatment modalities for intracranial AVMs include microsurgical treatment, endovascular embolisation, radiosurgical treatment and combination of two or three of the above. The risks associated with AVM treatment must be weighed against the natural history of haemorrhage. ${ }^{10} 11$ However, the natural history of intracranial AVMs is still largely unknown. ${ }^{11}$ Microsurgery offers the highest immediate cure rate. ${ }^{11}$ However, microsurgical resection of intracranial AVMs, especially AVMs in eloquent locations, remains challenging and carries high risk of complications.

Currently, there are mainly two AVM grading systems that are commonly used in clinical practice: the Spetzler-Martin grading system and the supplementary grading system. The Spetzler-Martin grading system was first introduced by Spetzler and Martin in 1986 and consists of three factors: AVM size, location (eloquent or non-eloquent) and patterns of venous drainage. ${ }^{12}$ The supplementary grading system was first proposed by Lawton et $a l^{13}$ in 2010 and embodies three factors: 
patient age, bleeding and diffuseness. Both the grading systems are well designed in assessing the risks associated with AVM surgery. However, other factors may also influence the treatment outcomes besides the above six factors in the two grading systems. ${ }^{11}$

Functional MRI (fMRI) is a new imaging technique to identify the functional imaging of eloquent area of the brain. Diffusion tensor imaging (DTI) is a potential technique to map eloquent fibre tracts. Until now, in assessing the surgical risk of AVMs, there is no predictive model based on multimodal fMRI. Small sample studies have reported that multimodal MRI techniques (fMRI, DTI and time-of-flight MR angiography (TOF-MRA)) are valuable tools for preoperative evaluation and treatment planning of brain AVMs. ${ }^{14-24}$ In our previous studies of AVMs based on multimodal imaging techniques, we have found that the least distance from the AVM nidus to the eloquence (the activated cortex or the eloquent fibre tracts) on fMRI and DTI studies is associated with surgical outcomes. ${ }^{25-28}$ A $5 \mathrm{~mm}$ distance from the eloquent fibres may be a suitable safety margin for postoperative function preservation. ${ }^{26-28}$ Based on our previous findings, we will create as well as validate a multimodal MRI technique-based predictive model to assess the surgical risk in each patient with AVM.

\section{STUDY OBJECTIVE}

The key purpose of this study is to create and validate a new predictive model for the surgical risk of intracranial AVMs. Based on this predictive model, we will propose a new supplementary grading system for intracranial AVMs.

\section{METHODS AND ANALYSIS}

\section{Study designs}

The study consists of two parts: the discovery set and the validation set. The discovery set is a retrospective analysis of 201 eligible patients with AVM from our brain AVM database of a prospective randomised controlled clinical trial-FMRINAVMS (fMRI Navigation in Intracranial Arteriovenous Malformation Surgery; ClinicalTrials.gov Identifier: NCT01758211) at Beijing Tiantan Hospital (China National Clinical Research Center for Neurological Diseases) between September 2012 and September $2015 .^{29}$ The validation set is a prospective cohort study of patients with AVM who will receive surgical resection for their AVMs at five major neurosurgical centres in China between June 2016 and June 2019. The study design is presented in figure 1 .

\section{Study participants}

Patient demographic characteristics (age, sex, history of haemorrhage and preoperative functional status), AVM features (size, location, arterial supply, patterns of venous drainage, Spetzler-Martin grade and diffuseness) and the lesion to eloquence distance (LED) obtained by multimodal MRI techniques. The LED means the least distance from the AVM to the eloquent cortex or the

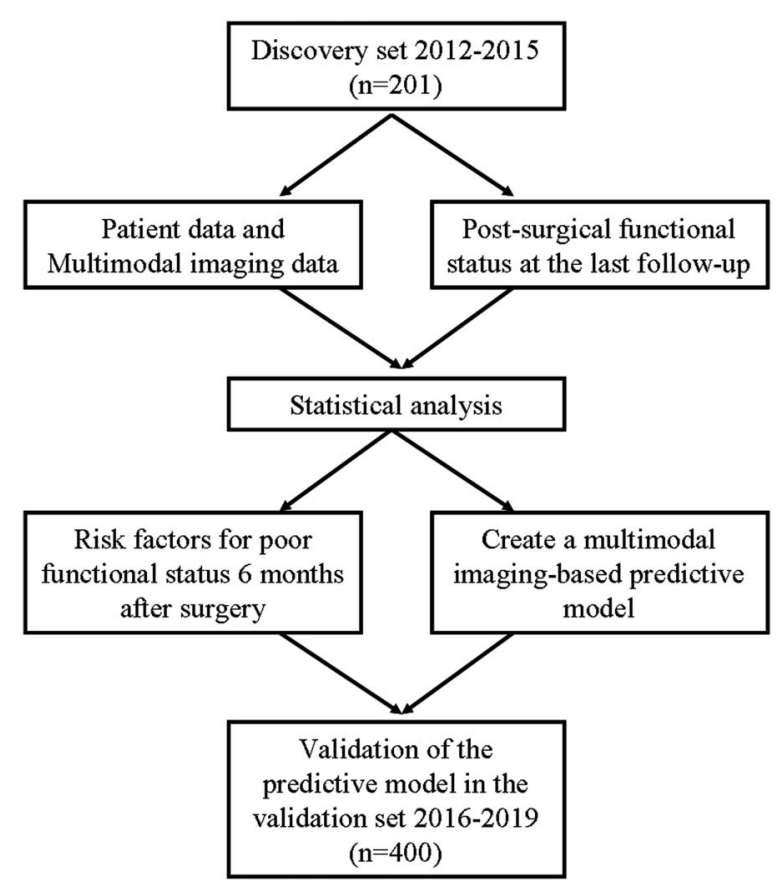

Figure 1 Diagram of the study protocol.

eloquent fibre tracts. Patient's functional status was or will be measured by the modified Rankin Scale (mRS) score. All patients in this study should meet the inclusion and exclusion criteria. Since the patients in the retrospective phase were chosen from the database of a prospective randomised controlled trial, written informed consent has already been obtained from the patients or their relatives. ${ }^{29}$ For the prospective study part (validation set), informed written consent should be obtained from eligible adult patients or from the guardians of eligible paediatric patients. All patients in the prospective part of this study can withdraw at any time.

\section{Inclusion criteria}

1. Patients aged between 12 and 60 years.

2. Patients with a definite diagnosis of AVM confirmed by preoperative digital subtraction angiography (DSA).

3. Patients with complete multimodal MRIs: preoperative structural MRI, blood oxygen level-dependent (BOLD)-fMRI, TOF-MRA, and DTI of the eloquent fibre tracts.

4. Patients opting for surgical management of their AVMs.

5. Patients without any treatment (microsurgery, radiosurgery, endovascular embolisation, or multimodality treatment) before enrolment.

\section{Exclusion criteria}

1. Patients receiving emergency surgery due to acute intracranial haematoma and resultant brain hernia.

2. Patients experiencing an AVM-related haemorrhage in the month before admission.

3. Patients without BOLD-fMRI and DTI data. 
4. Patients with severe diseases that prevent them from microsurgical treatment.

5. Patients without written informed consent.

\section{Multimodal MRI data acquisition}

Structural MRIs, BOLD-fMRI, diffusion tensor imaging (DTI) and TOF-MRA were or will be performed on a 3T Siemens Tim Trio MRI (Siemens Healthcare, Erlangen, Germany) as previously described. ${ }^{22} \quad 24 \quad 30 \quad 31$ For BOLD-fMRI, maps of neural activity within motor, language or visual cortex were or will be generated as described in our previous publication. ${ }^{29}$ The preoperative MRI data were or will be saved as DICOM format. The data were or will be transferred to an offline iPlan V.3.0 workstation (BrainLab, Feldkirchen, Germany) for analysis. The three-dimensional (3D) model of the eloquent cortex (motor, language or visual), the fibre tracking of the eloquent fibres (corticospinal tract, the arcuate fasciculus or the optic radiation) and the $3 \mathrm{D}$ anatomic structure of the AVMs were or will be reconstructed as described in our previous publications. ${ }^{26-28} 3233$

AVM angioarchitecture (including the feeding artery, the nidus and the venous drainage) and AVM diffuseness will be acquired by the 3D MRA. The maximum diameter of the AVMs was or will be measured from the structural MRI on the axial, coronary and sagittal directions. The LEDs (the least distances from AVM to the eloquent cortex and eloquent fibres) were or will be measured respectively on the axial, coronary and sagittal directions on 3D reconstruction of the AVM, the eloquent cortex and the eloquent fibre. All the multimodal MR data from different centres will be collected by two neuroradiologists.

\section{Treatment procedure}

Preoperative multimodality MRI reconstruction data (reconstruction of BOLD-fMRI, DTI and TOF-MRA) were or will be displayed on a Kolibri WS V.2.0 navigation system (BrainLab, Germany) during surgery. Craniotomy was or will be performed according to the preoperative plan based on the multimodal reconstruction data on the navigation system. After craniotomy, the feeding arteries, AVM nidus and draining veins of the AVM as well as adjacent eloquent cortex or eloquent fibres were or will be identified with the navigator's probe, as described in our previous publications. ${ }^{29} 32$

All microsurgical techniques or equipment such as intraoperative ultrasound scanner and indocyanine fluorescence angiography were or will be available during surgical resection. Motor evoked potential and somatosensory evoked potential were or will be provided as needed for intraoperative monitoring. ${ }^{29} 32$

\section{Outcome evaluation}

For the retrospective phase, a nurse clinician, under the supervision of a neurologist, performed the outcome evaluation at presentation and at the last follow-up through telephone or patient's routine clinical visit. For the prospective phase, patient outcome will be assessed by a physician or a nurse clinician from each of the five participating centres. All the physicians or nurse clinicians have been professionally trained in evaluating patient outcomes (including using the mRS) before the study.

For the retrospective phase, we will analyse the change of functional status (mRS score) between two time points: at pretreatment and at the final follow-up. For the prospective phase, the primary outcome is the change of mRS score between two time points: at pretreatment and 6 months after microsurgery. Good outcome is defined as a final mRS score of $0-2$ and poor outcome is defined as a final $\mathrm{mRS}>2$. Improved outcome is defined as a decreased or unchanged mRS score (mRS 6 months after surgery minus pretreatment $\mathrm{mRS} \leq 0)$. Worsened outcome is defined as an increased mRS score (mRS 6 months after surgery minus pretreatment $\mathrm{mRS} \leq 0$ ). The potential influencing factors for worsened outcome will be collected. These factors are patient's age, history of haemorrhage, preoperative mRS, AVM size, AVM location, deep arterial perforator supply, patterns of venous drainage, diffuseness and the LED on multimodal MRIs.

For the prospective phase, the second outcomes include AVM obliteration, headache and seizure outcome, surgical complications, and neurological deficits (motor, language or visual deficits). AVM obliteration will be assessed by postoperative DSA (3-7 days after surgery). Seizure outcome will be assessed using the modified Engel scale (four classes). The surgical complications include haemorrhagic stroke, intracranial infection, de novo seizures and cerebral infarction from the surgical date to 6 months after surgery. Motor deficits will be assessed using muscle strength measured by muscle strength grading scale. Language deficits will be measured by Western Aphasia Battery (WAB) 6 months after surgery. Visual field deficits will be measured by visual field testing 6 months after surgery.

\section{Sample size}

The retrospective phase of the study includes 201 eligible patients with AVM from our brain AVM database of a prospective randomised controlled clinical trialFMRINAVMS (ClinicalTrials.gov Identifier: NCT01758211) at Beijing Tiantan Hospital (China National Clinical Research Center for Neurological Diseases) between September 2012 and September 2015. We will use the data of these 201 patients to create the new predictive model. From June 2016 to June 2019, we will enrol 400 eligible patients from five neurosurgical centres with highvolume AVM practices and high-level neurosurgical expertise. The new model created from the retrospective phase will be validated in the prospective 400 eligible patients.

Statistical analyses to create a new predictive model Statistical analyses will be performed using the Statistical Package for the Social Sciences software (V.20.0; SPSS, 
Chicago, Illinois, USA). Patient characteristics and AVM features based on multimodal imaging will be summarised using descriptive statistics for continuous variables and categorical variables. These variables include patient demographics (age, sex, history of haemorrhage and pretreatment mRS scores), AVM features (size, cortical or deep location, eloquent or non-eloquent location, arterial supply, patterns of venous drainage, diffuseness and associated aneurysm) and multimodal imaging data (the least distance from the AVM to the eloquent cortex or to the eloquent fibres based on 3D reconstruction).

For the 201 patients in the discovery set, to create a predictive model for worsened neurological outcome, we will analyse patient characteristics, AVM features and multimodal MRI data by univariate and multivariate logistic regression analyses using worsened outcome as the binary response. To assess the predictive ability of the multivariate model, we measured the area under the receiver operating characteristic (AUROC) curve based on the predicted values in the multivariate analyses. We will also measure the AUROC for Spetzler-Martin grading system and the supplementary grading system models. Comparison between the new predictive model and the current two grading system models will be performed based on AUROC. The multimodal MRI-based predictive model then will be created. According to our preliminary result, haemorrhagic presentation $(\mathrm{H})$, diffuseness (D), deep venous drainage (V) and the LED (L) on multimodal MRI were independent predictors of worsened outcomes after AVM surgery. The predictive accuracy of the HDVL (Haemorrhagic presentation, Diffuseness, deep Venous drainage and LED) model is superior to the Spetzler-Martin grading system and the supplemented S-M grading system. The HDVL grading system will be proposed.

\section{Validation of the new predictive model}

For the patients (a sample size of 400 eligible patients) in the multicentre prospective cohort study, the new predictive model will be verified in predicting poor outcomes. In the validation set, the new predictive model will also be compared with the current two existing grading systems by using the AUROC to predict worsened neurological outcomes. If the new predictive model is superior to the Spetzler-Martin grading system and the supplementary grading system, the new model is validated and a new grading system will be proposed.

\section{Data management}

All data in the discovery set of 201 patients can be collected from our prospectively maintained AVM database at Beijing Tiantan Hospital. All data in the validation set will be prospectively collected using an electronic case report form through a study website. Five major neurological centres in China can access and manage patient information by login and password. All patients enrolled will be carefully monitored until 6 months after surgery.
Data safety, data quality, monthly auditing and statistical analysis will be managed by a third party-SUN HEALTH (Beijing), who shall be responsible for notifying any issues that may arise during the whole cohort study. The whole study will be supervised by Beijing Municipal Science and Technology Commission and China National Clinical Research Center for Neurological Diseases. Any issue occurring during the cohort study will be reported to these two oversight authorities. Recommendations from these two authorities will be forwarded to the principal investigators to balance the risk and benefit. The oversight authorities have the right to terminate the study if great risk occurs during the study.

\section{Duration of the study}

The discovery set includes 201 eligible patients with AVM who were surgically treated in Beijing Tiantan Hospital between September 2012 and September 2015. The validation set will enrol 400 eligible patients with AVM who will receive microsurgical treatment in five major neurosurgical centres in China from June 2016 until June 2019. The first participant was recruited for the prospective phase of the study on 3 June 2016.

\section{ETHICS AND DISSEMINATION}

The study is supported by Key Project of Beijing Municipal Science and Technology Commission (grant number D161100003816006). The first part of this study is a retrospective review of patients treated at one centre (Beijing Tiantan Hospital). The second part of this study is a prospective cohort study of patients treated at five neurosurgical centres. To create and validate the multimodal fMRI-based predictive model for AVM surgery, all patients should have complete preoperative fMRI data and meet the inclusion and exclusion criteria. This study will be conducted in accordance with ethical principles of the Declaration of Helsinki and the Good Clinical Practice guidelines of the International Conference on Harmonisation. Written informed consent will be obtained from each adult participant or from the guardian of each paediatric participant in the validation set. SW is the principal investigator of this cohort study. Data collection, statistical analysis, interpretation and dissemination of study results will be managed under his direct supervision. The final results of this study will be disseminated through printed media in December 2019.

\section{DISCUSSION}

In 1986, Spetzler and Martin introduced the first AVM grading system (the Spetzler-Martin grading system) to estimate the risk of AVM surgery. ${ }^{12}$ The Spetzler-Martin grading system is a five-point scale that is based on AVM size, eloquent or non-eloquent AVM location, and patterns of venous drainage. According to S-M grading system, patients with asymptomatic grade IV and V AVMs 
should not be surgically treated due to the high risk of surgical complications. ${ }^{12}$ However, this standpoint is not universally accepted. Based on the original five-tier Spetzler-Martin grading system, Spetzler and Ponce proposed a three-tier classification for AVMs in 2011. ${ }^{34}$ In this three-tier classification, AVMs are divided into class A (S-M grade I and II AVMs), class B (S-M grade III AVMs) and class $\mathrm{C}$ (S-M grade IV and V AVMs). This three-tier classification simplifies treatment recommendations. However, observation is still recommended for patients with asymptomatic class C (S-M grade IV and V) AVMs. ${ }^{34}$

In 2010, Lawton et $a l^{13}$ proposed a supplementary grading system including three additional parameters that may affect surgical outcomes: patient age, haemorrhagic presentation and nidal diffuseness. The authors proposed a full multivariable model (combination of S-M grading system and supplementary system) and a supplementary model. In the analysis of 1009 patients, the authors found that the full multivariable model had the highest predictive accuracy. ${ }^{35}$ However, surgical selection for patients with AVM remains challenging. Just as described in a recent review in Neurosurgery, future grading scales may incorporate imaging features. ${ }^{11}$ Multimodal MRIs have proved to be a valuable tool in preoperative evaluation and surgical planning. In our clinical practice, we have found that the least distance from AVM to eloquent cortex or eloquent fibres based on multimodal imaging is significantly associated with surgical outcomes. ${ }^{25-28}$ Based on our previous studies, we have designed this study to propose a multimodal MRI-based predictive model for surgical outcomes of intracranial AVMs. We will compare the predictive accuracy between the new predictive model and the current two grading systems. We hypothesise that the new predictive model in this study has the highest predictive accuracy.

Contributors SW has obtained research funding and is the principal investigator of the study. $\mathrm{YC}, \mathrm{YZ}$ and $\mathrm{SW}$ have developed this study protocol. $\mathrm{XT}, \mathrm{JW}, \mathrm{YC}, \mathrm{YZ}$ and SW have participated in the final design of the study.

Funding This work is supported by the Key Project of Beijing Municipal Science and Technology Commission (grant number D161100003816006).

Competing interests None declared.

Ethics approval The Institutional Review Board of Beijing Tiantan Hospital Affiliated to Capital Medical University.

Provenance and peer review Not commissioned; externally peer reviewed.

Open Access This is an Open Access article distributed in accordance with the Creative Commons Attribution Non Commercial (CC BY-NC 4.0) license, which permits others to distribute, remix, adapt, build upon this work noncommercially, and license their derivative works on different terms, provided the original work is properly cited and the use is non-commercial. See: http:// creativecommons.org/licenses/by-nc/4.0/

\section{REFERENCES}

1. Abecassis IJ, Xu DS, Batjer $\mathrm{HH}$, et al. Natural history of brain arteriovenous malformations: a systematic review. Neurosurg Focus 2014;37:E7.

2. ApSimon $\mathrm{HT}$, Reef $\mathrm{H}$, Phadke RV, et al. A population-based study of brain arteriovenous malformation: long-term treatment outcomes. Stroke 2002;33:2794-800.
3. Ondra SL, Troupp H, George ED, et al. The natural history of symptomatic arteriovenous malformations of the brain: a 24-year follow-up assessment. J Neurosurg 1990;73:387-91.

4. Stapf C, Mast H, Sciacca RR, et al. Predictors of hemorrhage in patients with untreated brain arteriovenous malformation. Neurology 2006;66:1350-5.

5. Tong X, Wu J, Lin F, et al. Risk factors for subsequent hemorrhage in patients with cerebellar arteriovenous malformations. World Neurosurg 2016;92:47-57.

6. Al-Shahi R, Bhattacharya JJ, Currie DG, et al. Prospective, population-based detection of intracranial vascular malformations in adults: the Scottish Intracranial Vascular Malformation Study (SIVMS). Stroke 2003;34:1163-9.

7. Andreussi L, Dhellemmes P. [Cerebral arteriovenous malformations. Natural history. Therapeutic approaches]. Agressologie 1990;31:237-9.

8. Niazi TN, Klimo PJr, Anderson RC, et al. Diagnosis and management of arteriovenous malformations in children. Neurosurg Clin N Am 2010;21:443-56.

9. Yamada S, Brauer FS, Colohan AR, et al. Concept of arteriovenous malformation compartments and surgical management. Neurol Res 2004;26:288-300.

10. Kim H, McCulloch CE, Johnston SC, et al. Comparison of 2 approaches for determining the natural history risk of brain arteriovenous malformation rupture. Am J Epidemiol 2010;171:1317-22.

11. Bendok BR, El Tecle NE, El Ahmadieh TY, et al. Advances and innovations in brain arteriovenous malformation surgery. Neurosurgery 2014;(74 Suppl 1):S60-73.

12. Spetzler RF, Martin NA. A proposed grading system for arteriovenous malformations. J Neurosurg 1986;65:476-83.

13. Lawton MT, Kim H, McCulloch $\mathrm{CE}$, et al. A supplementary grading scale for selecting patients with brain arteriovenous malformations for surgery. Neurosurgery 2010;66:702-13.

14. Baumann SB, Noll DC, Kondziolka DS, et al. Comparison of functional magnetic resonance imaging with positron emission tomography and magnetoencephalography to identify the motor cortex in a patient with an arteriovenous malformation. J Image Guid Surg 1995;1:191-7.

15. Stapleton SR, Kiriakopoulos E, Mikulis D, et al. Combined utility of functional MRI, cortical mapping, and frameless stereotaxy in the resection of lesions in eloquent areas of brain in children. Pediatr Neurosurg 1997;26:68-82.

16. Lazar RM, Marshall RS, Pile-Spellman J, et al. Interhemispheric transfer of language in patients with left frontal cerebral arteriovenous malformation. Neuropsychologia 2000;38:1325-32.

17. Seghier M, Lazeyras F, Momjian S, et al. Language representation in a patient with a dominant right hemisphere: fMRI evidence for an intrahemispheric reorganisation. Neuroreport 2001;12:2785-90.

18. Ozdoba C, Nirkko AC, Remonda L, et al. Whole-brain functional magnetic resonance imaging of cerebral arteriovenous malformations involving the motor pathways. Neuroradiology 2002;44:1-10.

19. Cannestra AF, Pouratian N, Forage J, et al. Functional magnetic resonance imaging and optical imaging for dominant-hemisphere perisylvian arteriovenous malformations. Neurosurgery 2004;55:804-12; discussion 12-4.

20. Thickbroom GW, Byrnes ML, Morris IT, et al. Functional MRI near vascular anomalies: comparison of cavernoma and arteriovenous malformation. $J$ Clin Neurosci 2004;11:845-8.

21. Ulmer JL, Hacein-Bey L, Mathews VP, et al. Lesion-induced pseudo-dominance at functional magnetic resonance imaging: implications for preoperative assessments. Neurosurgery 2004;55:569-79; discussion 80-1.

22. Stancanello J, Cavedon C, Francescon P, et al. BOLD fMRI integration into radiosurgery treatment planning of cerebral vascular malformations. Med Phys 2007;34:1176-84.

23. Caramia F, Francia A, Mainero C, et al. Neurophysiological and functional MRI evidence of reorganization of cortical motor areas in cerebral arteriovenous malformation. Magn Reson Imaging 2009;27:1360-9.

24. Lee L, Sitoh YY, Ng I, et al. Cortical reorganization of motor functional areas in cerebral arteriovenous malformations. J Clin Neurosci 2013;20:649-53.

25. Jiao $\mathrm{Y}$, Lin $\mathrm{F}, \mathrm{Wu} \mathrm{J}$, et al. Lesion-to-eloquent fibre distance is a crucial risk factor for pre-surgical evaluation of arteriovenous malformations in temporo-occipital junctions. World Neurosurg 2016;93:355-64.

26. Lin F, Zhao B, Wu J, et al. Risk factors for worsened muscle strength after the surgical treatment of arteriovenous malformations of the eloquent motor area. J Neurosurg 2016;125:289-98. 
27. Lin F, Wu J, Zhao B, et al. Preoperative functional findings and surgical outcomes in patients with motor cortical arteriovenous malformation. World Neurosurg 2016;85:273-81.

28. Tong $\mathrm{X}, \mathrm{Wu} \mathrm{J}$, Lin $\mathrm{F}$, et al. Visual field preservation in surgery of occipital arteriovenous malformations: a prospective study. World Neurosurg 2015;84:1423-36.

29. Zhao B, Cao Y, Zhao Y, et al. Functional MRl-guided microsurgery of intracranial arteriovenous malformations: study protocol for a randomised controlled trial. BMJ Open 2014;4:e006618.

30. Henriksson L, Raninen A, Näsänen R, et al. Training-induced cortical representation of a hemianopic hemifield. $J$ Neurol Neurosurg Psychiatr 2007;78:74-81.

31. Sanjuán A, Bustamante JC, Forn C, et al. Comparison of 2 fMR tasks for the evaluation of the expressive language function. Neuroradiology 2010;52:407-15.
32. Lin F, Jiao $\mathrm{Y}, \mathrm{Wu} \mathrm{J}$, et al. Effect of functional MRI-guided navigation on surgical outcomes: a prospective controlled trial in patients with arteriovenous malformations. J Neurosurg 2016;1:1-10.

33. Lin F, Wu J, Wang L, et al. Surgical treatment of cavernous malformations involving the posterior limb of the internal capsule: utility and predictive value of preoperative diffusion tensor imaging. World Neurosurg 2016;88:538-47.

34. Spetzler RF, Ponce FA. A 3-tier classification of cerebral arteriovenous malformations: clinical article. J Neurosurg 2011;114:842-9.

35. Kim H, Abla AA, Nelson J, et al. Validation of the supplemented Spetzler-Martin grading system for brain arteriovenous malformations in a multicenter cohort of 1009 surgical patients. Neurosurgery 2015;76:25-31. 